NOTAS 



\title{
ANÁLISIS PROBABILÍSTICO DE LA PERSISTENCIA DE LOS DÍAS DE LLUVIA EN VILLENA (ALICANTE) DURANTE LA PRIMAVERA DE 2004
}

\author{
César Azorín Molina \\ Instituto Universitario de Geografía \\ Universidad de Alicante
}

\section{RESUMEN}

En este trabajo se aborda un breve análisis acerca del carácter extraordinariamente anómalo que presentó la precipitación en forma de lluvia durante la primavera de 2004. El estudio se centra en el examen detallado del grado de persistencia de este hidrometeoro en Villena (Alicante), en los meses de marzo, abril y mayo. Se emplean sencillos métodos de análisis probabilístico con objeto de verificar el estado de inercia de los días lluviosos, algo muy poco común en los climas de filiación mediterránea.

Palabras clave: lluvia, grado de persistencia, métodos probabilísticos, primavera 2004, Villena (Alicante).

\section{ABSTRACT}

This work consists of a brief analysis of the outstandingly abnormal character featured by rainfalls during spring 2004. This study focuses on the detailed examination of the persistence degree of this hydrometeor in Villena (Alicante), in March, April and May. Simple probabilistic analysis methods are used in order to check the state of inertia in rainy days, something which is not at all common in Mediterranean-type climates.

Key words: rain, degree of persistence, probabilistic methods, spring 2004, Villena (Alicante). 


\section{Introducción: Objetivos del trabajo}

El estado del tiempo atmosférico en un momento concreto (temperatura, humedad, precipitación, viento, presión, insolación, nubosidad, etc.), se encuentra relacionado con las condiciones meteorológicas registradas en el pasado más inmediato. Esta dependencia disminuye a medida que aumenta el tiempo transcurrido. En relación con ello, se entiende por inercia la capacidad que tiene un cuerpo a persistir o, lo que es lo mismo, la resistencia que opone éste a cambiar de un estado a otro. Esta propiedad resulta, en mayor o menor medida, inherente a todas las variables meteorológicas (Martín Vide, 2003), y depende directamente de la continuidad ${ }^{1}$ de cada parámetro atmosférico.

Esta condición de variable no continua para el caso de la precipitación, unida a otros objetivos, ha sido aprovechada por la comunidad de climatólogos españoles, así como por otros investigadores de ciencias afines, para estudiar el grado de persistencia de la precipitación en forma de lluvia y, sobre todo, cuantificar la longitud y recurrencia de las secuencias secas, trabajos que se han enfocado principalmente a la fachada oriental de la península Ibérica; De enorme utilidad de cara a la gestión de los recursos hídricos y planificación de los usos del suelo en el territorio (Gil Olcina y Morales Gil, 2001), cuestión que resulta si cabe más importante si además estos estudios se acompañan de un análisis de la variabilidad natural de la persistencia de la precipitación en forma de lluvia (Fernández de Arróyabe Hernáez, 2002). Trabajos que han seguido idéntico enfoque han sido desarrollados desde mediados del siglo pasado ${ }^{2}$, destacando la aplicación de la técnica probabilística markoviana (modelo estocástico de las cadenas de Markov) de Domínguez (1973), Martín Vide (1981), Raso Nadal (1982), Martín Vide (1983), Martín Vide y Moreno García (1985), Martín Vide et al. (1989), Conesa García y Martín Vide (1993), Barriendos Vallvé y Martín Vide (1996), Gómez Navarro (1996), Gómez Navarro (1997), Lana y Burgueño (1998), Gómez Navarro et al. (2000), Luengo Ugidos et al. (2002), así como aplicados al estudio de la concentración diaria de la precipitación (Martín Vide, 2004), entre otros muchos estudios.

Por medio de esta nota no se pretende justificar los elevados totales de precipitación registrados en los observatorios meteorológicos del sureste de España en los meses de primavera de 2004. En este sentido, este estudio no entra a valorar de lleno la cuantía de precipitación caída durante la pasada primavera, sino que, fuera de esto último, se centra en verificar, mediante el empleo de métodos probabilísticos muy sencillos, el carácter repetido de un estado meteorológico poco común en los climas surestinos de filiación mediterránea; La persistencia de los días de lluvia.

En el recuerdo y opinión de la población queda el haber vivido una primavera de ambiente atmosférico muy destemplado, de tiempo fresco, lluvioso y de falta de días soleados; Algo anormal ya que, como se conoce, la elevada fracción de insolación representa el denominador común en las condiciones del clima de la región del sur peninsular³.

1 Por vía de ejemplo, la temperatura del aire presenta un carácter de persistencia mayor en comparación a la precipitación, al tratarse de una variable física continua; Frente a la segunda que no lo es (Martín Vide, 2003: 53-54).

2 Volcados hacia el análisis del comportamiento secuencial de la precipitación (rachas secas y lluviosas) para distintas áreas y escalas de trabajo.

3 Los valores termométricos máximos no superaron la marca de los $30^{\circ} \mathrm{C}$ en los observatorios meteorológicos alicantinos hasta los días 28 ó 29 de mayo, algo completamente atípico para estas latitudes teniendo en cuenta la época del año. 


\section{Datos climáticos y metodología}

\subsection{Datos de precipitación}

El estudio de la persistencia del hidrometeoro de la lluvia se ha realizado utilizando los datos de precipitación recogidos en las fichas de anotación diaria de la estación meteorológica propia instalada en el centro de la ciudad de Villena (Alicante), correspondientes a los meses de primavera de marzo, abril y mayo de 2004 (vid. Tabla 6, en el apartado de anexo final) y a la serie pluviométrica quinquenal 1999-2003. A efectos prácticos, se ha considerado como día de lluvia a toda aquella jornada en la que existía anotación de precipitación, incluyendo los episodios de lluvia inapreciable (ip.), al entender éstas como jornadas que estuvieron gobernadas por condiciones atmosféricas de inestabilidad.

Los datos de precipitación manejados y los resultados alcanzados en este estudio climático pueden ser extrapolados, teniendo en cuenta que éstos son representativos de un comportamiento de carácter general, al resto del territorio de la provincia de Alicante.

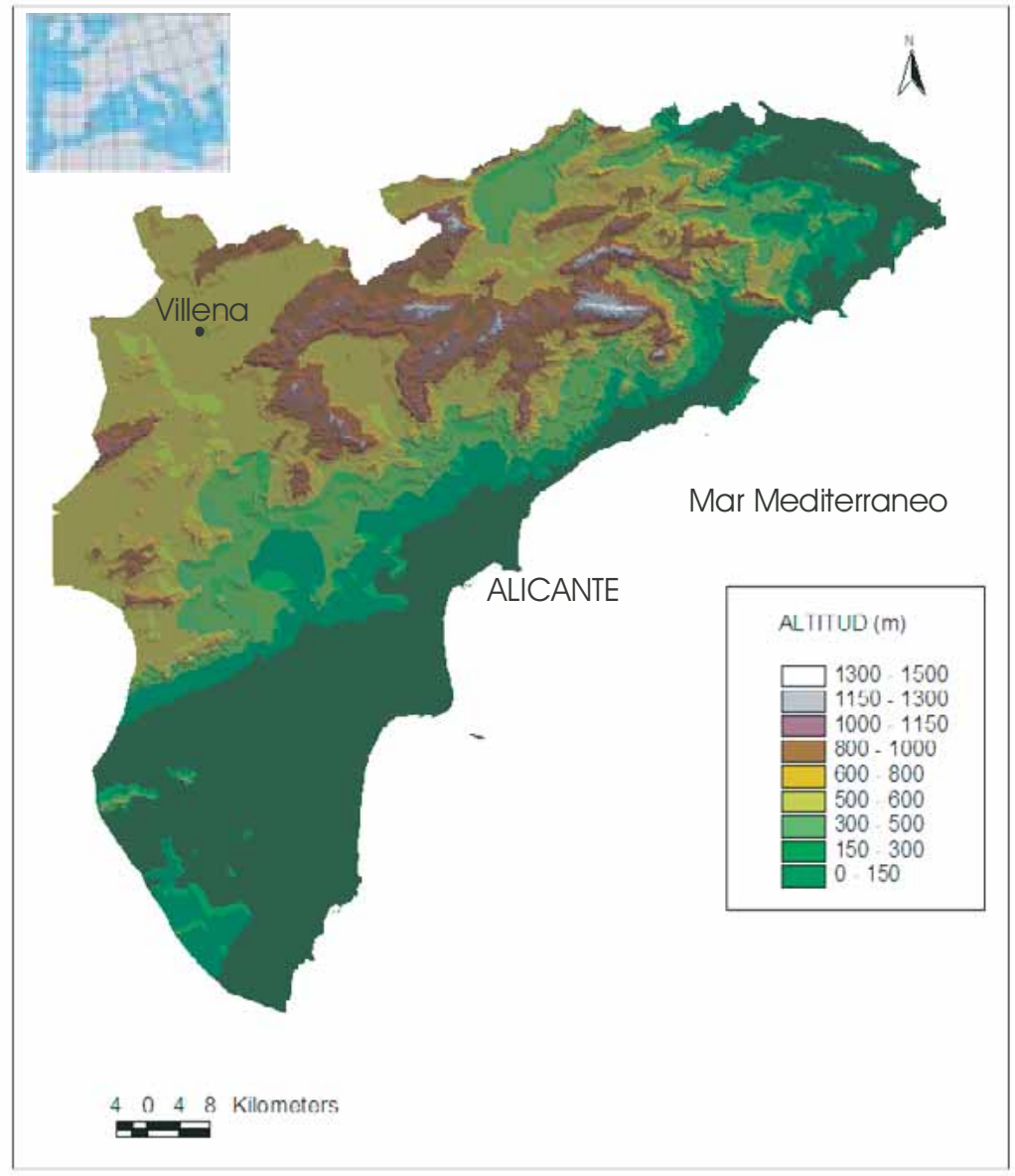

MAPA 1. Localización de la estación meteorológica de Villena en el área de estudio. 


\subsection{Métodos de análisis probabilístico}

Con objeto de valorar la inercia a persistir de los días lluviosos en Villena durante los meses de primavera de marzo, abril y mayo de 2004, se han aplicado sencillos test de análisis estadístico, que se presentan en el siguiente subepígrafe. Los procedimientos estadísticos calculados para estimar el grado de persistencia de los días lluviosos han sido los siguientes:

- Número absoluto de rachas lluviosas $(N R)$, mensual y estacional.

- Longitud máxima y mínima absoluta, y media $\left(l_{l}\right)$, de las secuencias de días lluviosos.

- Coeficientes de persistencia.

Probabilidad de día de lluvia $\left(p_{I}\right)$.

Probabilidad de día sin lluvia $\left(p_{0}\right)$.

Probabilidad de día de lluvia después de día de lluvia $\left(p_{11}\right)$.

Constatación del índice de persistencia de los días de lluvia.

\subsubsection{Método de las rachas}

Una racha o secuencia es un período de tiempo constituido por días que presentan un mismo carácter en el estado meteorológico (Martín Vide, 2003); En el caso de estudio, jornadas consecutivas registrando precipitación en forma de lluvia. La aplicación de este método consiste en contabilizar el número absoluto de rachas o secuencias de días de lluvia durante el período de estudio, así como cuantificar la longitud máxima y mínima absoluta de las mismas. Para ello, previamente se deben haber agrupado las jornadas de lluvia en rachas. Cuando una secuencia de días de lluvia se prolonga entre dos meses ${ }^{4}$, se ha procedido a asignar la racha a aquel mes que agrupaba un mayor número de jornadas con precipitación líquida (Douguédroit, 1987).

El cálculo de la longitud media de las rachas de lluvia $\left(l_{I}\right)$ se obtiene como cociente entre el total de días de lluvia $(L)$ y el total de rachas $(N R)$ del período de estudio considerado.

$$
l_{1}=L / N R
$$

\subsubsection{Coeficientes de persistencia}

El estudio de la inercia de un estado meteorológico - persistencia de los días lluviosos se realiza mediante el empleo de coeficientes de persistencia (Martín Vide, 2003). En este trabajo se han utilizado tres procedimientos estadísticos de cálculo matemático sencillo:

- Probabilidad de día de lluvia $\left(p_{l}\right)$ : Cociente entre el número de días de lluvia $(L)$ y el número de días observados $(N)$.

$$
p_{l=} L / N
$$

- Probabilidad de día sin lluvia o seco $\left(p_{0}\right)$ : Cociente entre el número de días sin lluvia o secos $(S)$ y el número de días observados $(N)$, o complementaria de $p_{1}$.

$$
p_{0=} S / N \quad \text { ó } \quad 1-p_{1}
$$

4 En el caso de estudio, entre los meses de abril y mayo (vid. Tabla 2). 
- Probabilidad de día de lluvia después de día de lluvia $\left(p_{l l}\right)$ - probabilidad condicionada - : Cociente entre el número de días de lluvia después de día de lluvia $\left(L^{\prime}\right)$ y el número de días lluviosos $(L)$.

$$
p_{11=} L^{\prime} / L
$$

- Índice de persistencia de los días lluviosos: Se cumple cuando la probabilidad condicionada de día de lluvia después de día de lluvia $\left(p_{I I}\right)$ es mayor al valor obtenido de probabilidad de día de lluvia $\left(p_{l}\right)$.

$$
p_{11}>p_{1}
$$

\section{Resultados}

\subsection{Balance pluviométrico de la primavera de 2004}

A pesar de que dentro de los objetivos principales del presente estudio no se ha concedido importancia a la justificación de los elevados registros pluviométricos medidos durante la primavera de 2004, reservamos este apartado para realizar un breve balance del comportamiento pluviométrico de los meses de marzo, abril y mayo en la zona de estudio (Villena).

Como rasgo general, destacó sobremanera las cantidades de precipitación registradas en cada uno de los tres meses, puesto que éstas superaron de forma notable la precipitación media mensual correspondiente al treinteno internacional 1961-1990, tomando como referencia los datos pluviométricos medios de la estación meteorológica oficial de la finca «La Vereda ${ }^{5}$, dependiente del Instituto Nacional de Meteorología (INM). De modo extraordinario lo hizo el mes de marzo, que acumuló 32,8 mm de superávit en relación a la media. En los meses de abril y mayo, los excedentes de agua precipitada resultaron también muy sig-

\section{Tabla 1}

CUADRO COMPARATIVO ENTRE LA PRECIPITACIÓN REGISTRADA DURANTE LA PRIMAVERA DE 2004 EN VILLENA-CIUDAD Y LA MEDIA EN LA ESTACIÓN OFICIAL DE «LA VEREDA» (VILLENA)

\begin{tabular}{|c|c|c|c|}
\hline \multirow{2}{*}{ Mes } & Villena-Ciudad & $\begin{array}{c}\text { «La Vereda» } \\
\text { (Villena) }\end{array}$ & \multirow{2}{*}{ (PP.1) - (PP.2) } \\
\cline { 2 - 3 } & $\begin{array}{c}\text { PP. PRI } \\
\text { PP. PRI 2004 } \\
\text { (PP.1) }\end{array}$ & $\begin{array}{c}\text { Media 1961-1990 } \\
\text { (PP.2) }\end{array}$ & \\
\hline Marzo & 60,3 & 27,5 & 32,8 \\
\hline Abril & 51,5 & 31,6 & 19,9 \\
\hline Mayo & 62,2 & 39,5 & 22,7 \\
\hline Total & $\mathbf{1 7 4}$ & $\mathbf{9 8 , 6}$ & $\mathbf{7 5 , 4}$ \\
\hline
\end{tabular}

Fuente: Observatorio meteorológico propio (Villena-Ciudad) y Atlas Climático de la Comunidad Valenciana (1961-1990). COPUT. Generalitat Valenciana. Elaboración propia. PP.: precipitación en $\mathrm{mm}$ o $1 / \mathrm{m}^{2}$

5 Observatorio meteorológico trasladado en la actualidad a la casa «La Parrala», de la que toma nombre la estación. 
nificativos: la lluvia de abril superó la media en 19,9 mm y mayo en 22,7 mm. El cómputo total de precipitación elevó a 174 mm la lluvia recogida en primavera, es decir, 75,4 mm en comparación al valor medio normal.

En síntesis, el balance pluviométrico general para la primavera de 2004 se resume en que resultó muy húmeda en todo el territorio alicantino ${ }^{6}$ - también para buena parte del territorio peninsular ${ }^{7}-$, como demuestran los datos presentados (vid. Tabla 1).

\subsection{Número absoluto y longitud máxima, mínima y media de las secuencias de días lluviosos}

En la tabla 2 se presentan los resultados obtenidos tras agrupar los días de lluvia de los meses de primavera de 2004 en rachas o secuencias lluviosas. En este sentido, se ha contabilizado un total de 14 rachas lluviosas, repartidas 6 en el mes de abril y 4 en los meses de marzo y mayo. El número absoluto de rachas o secuencias lluviosas $(N R)$, si se analiza por separado, dice muy poco a la hora de valorar la persistencia del fenómeno de la lluvia, de tal modo que requiere ser comparado con otro dato: el total de días de lluvia $(L)$.

En consecuencia, si un número pequeño de rachas $(N R)$ agrupa a un elevado número de días de lluvia $(L)$, la persistencia de este hidrometeoro será mayor en comparación si lo hace un número menor de jornadas lluviosas. Para estimarlo, es imprescindible el cálculo de la longitud media de las secuencias de días de lluvia $\left(l_{l}\right)$. Así pues, si bien en los meses

Tabla 2

NÚMERO ABSOLUTO Y LONGITUD MÁXIMA Y MÍNIMA DE LAS RACHAS LLUVIOSAS EN VILLENA DURANTE LA PRIMAVERA DE 2004

\begin{tabular}{|c|c|c|}
\hline Mes & $\begin{array}{c}\text { Longitud de las rachas lluviosas } \\
\text { (en días) }\end{array}$ & $\begin{array}{c}\mathbf{N}^{\circ}{ }^{\circ} \text { de rachas lluviosas } \\
\text { (NR) }\end{array}$ \\
\hline Marzo & $1,2,4,7$ & 4 \\
\hline Abril & $1,6,2,1,1,9 \rightarrow$ & 6 \\
\hline Mayo & $5,3,3,1$ & 4 \\
\hline Resumen & $\begin{array}{c}\text { Máx. 9 días } \\
\text { Mín. 1 día }\end{array}$ & Total: $\mathbf{1 4}$ \\
\hline
\end{tabular}

Fuente: Observatorio meteorológico propio (Villena-Ciudad). Elaboración propia.

$\rightarrow$ Racha lluviosa prolongada entre el 28 de abril y 6 de mayo (vid. Anexo final. Tabla 6).

6 Hasta la llegada del verano, que marcó un hiato pluviométrico muy importante, la estación astronómica de primavera resultó extraordinariamente húmeda en todo el territorio alicantino. En conjunto, llovió un 50\% de la precipitación que se registra por término medio durante todo un año en los meses de marzo, abril y mayo. En el caso de la comarca de la Vega Baja del Segura, la más necesitada de agua, este valor porcentual se elevó a un 85\%: Por ejemplo, Pilar de la Horadada o Torrevieja, así como otras poblaciones vecinas, registraron en estos tres meses de primavera más de $240 \mathrm{~mm}$ de lluvia, cuando de por media (1961-1990) la precipitación apenas supera los 75 $\mathrm{mm}$. En estos mismos términos resultó la cuantía de lluvia recogida en Pinoso (238,2 mm), considerado como uno de los polos más secos de la provincia, así como los $455 \mathrm{~mm}$ registrados en Denia. Estas precipitaciones fueron muy beneficiosas para el campo de secano y para las reservas hídricas, aunque afectaron al cultivo de variedades de fruta temprana (níspero y cereza).

7 Así lo hizo constar el Instituto Nacional de Meteorología (INM) a través de un comunicado oficial haciendo balance, una vez finalizadas las tres primeras estaciones - otoño, invierno y primavera - , del año hidrometeorológico 2003-2004, contenida en el apartado de Noticias de la página web del INM a fecha 21/06/2004: «El año hidrometeorológico. En conjunto, llovió un 15\% más». En los meses de marzo, abril y mayo el volumen total de precipitaciones sobre España superó en torno a un $20 \%$ el valor medio, alcanzándose superávits de lluvias de algo más del $25 \%$ en los meses de marzo y mayo. Las precipitaciones fueron abundantes en el este y centro peninsular, y escasas hacia el noroeste. 


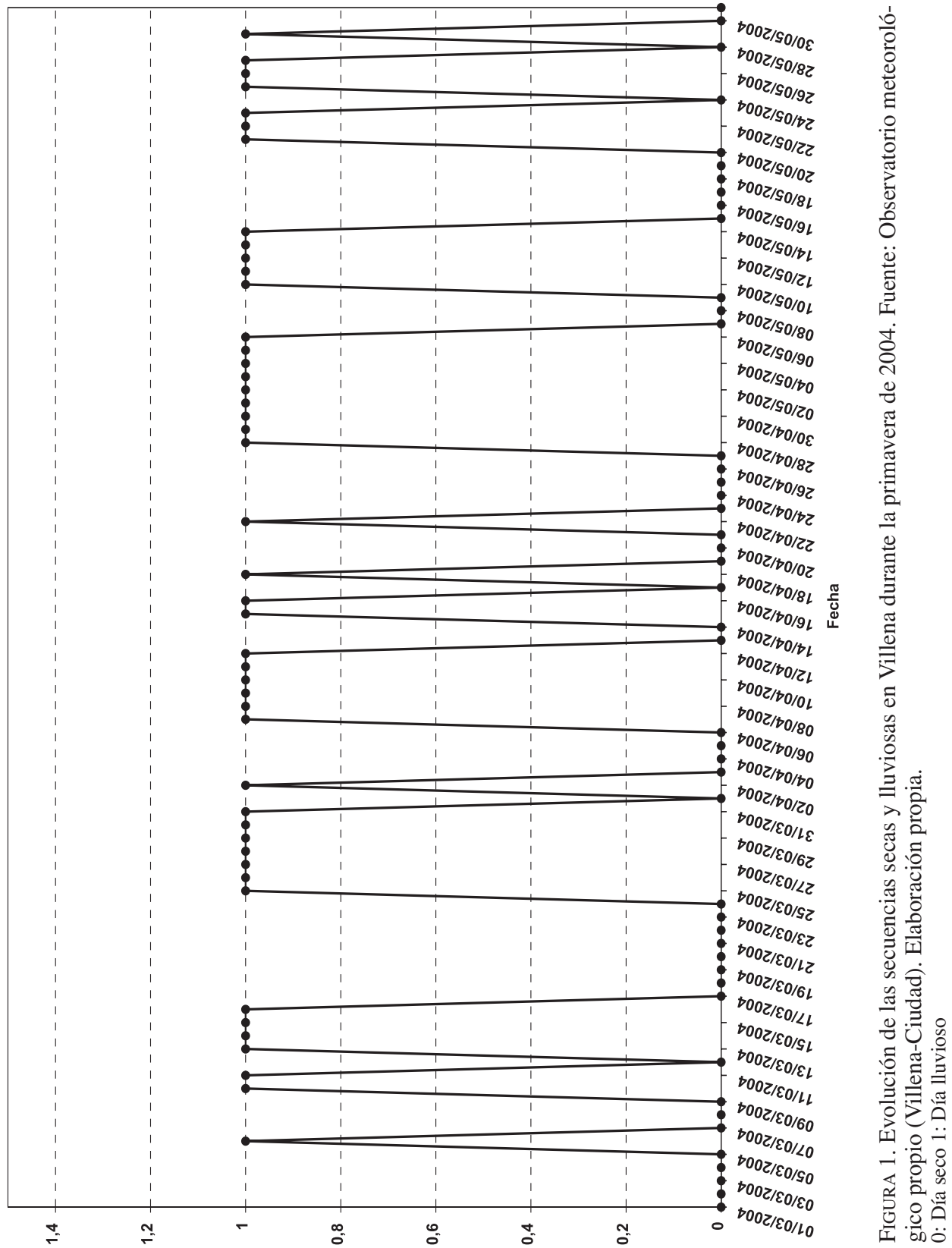




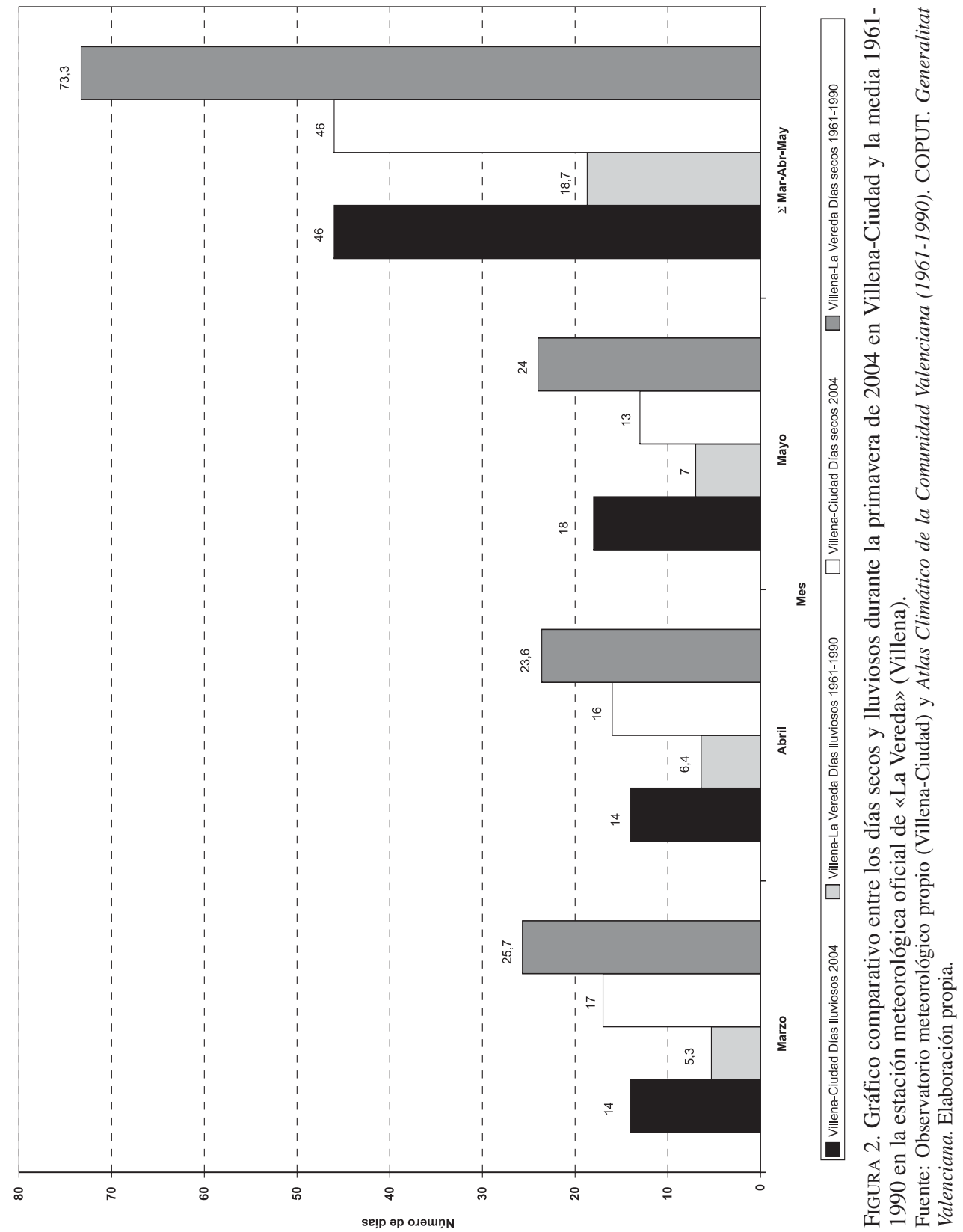


Tabla 3

LONGITUD MEDIA DE LAS SECUENCIAS LLUVIOSAS $\left(l_{1}\right)^{*}$ EN VILLENA DURANTE LA PRIMAVERA DE 2004

\begin{tabular}{|c|c|c|c|}
\hline Mes & $\begin{array}{c}\mathbf{N}^{{ }^{\mathbf{0}}} \text { días de lluvia } \\
(\mathbf{L})\end{array}$ & $\begin{array}{c}\mathbf{N}^{\mathbf{0}} \text { de rachas lluviosas } \\
(\mathbf{N R})\end{array}$ & $\begin{array}{c}\text { Longitud media de las } \\
\text { rachas de lluvia* }\left(\mathbf{l}_{\mathbf{1}}\right)\end{array}$ \\
\hline Marzo & 14 & 4 & 3,5 \\
\hline Abril & 14 & 6 & 2,33 \\
\hline Mayo & 18 & 4 & 4,5 \\
\hline Total & $\mathbf{4 6}$ & $\mathbf{1 4}$ & $\mathbf{3 , 2 9}$ \\
\hline
\end{tabular}

Fuente: Observatorio meteorológico propio (Villena-Ciudad). Elaboración propia.

* En días.

Tabla 4

LONGITUD MEDIA DE LA RACHAS DE LLUVIA $\left(l_{1}\right) *$ EN VILLENA DURANTE PRIMAVERA (SERIE QUINQUENAL 1999-2003)

\begin{tabular}{|c|c|c|c|c|}
\hline Año & Marzo & Abril & Mayo & Media Mar-May \\
\hline $\mathbf{1 9 9 9}$ & 2,3 & 1,5 & 1,2 & 1,6 \\
\hline $\mathbf{2 0 0 0}$ & 1,2 & 2 & 2,2 & 1,8 \\
\hline $\mathbf{2 0 0 1}$ & 1,5 & 1 & 1,9 & 1,5 \\
\hline $\mathbf{2 0 0 2}$ & 3 & 3,5 & 2,5 & 3 \\
\hline $\mathbf{2 0 0 3}$ & 2,7 & 1,7 & 2,7 & 2,2 \\
\hline $\begin{array}{c}\text { Media } \\
\text { Mensual }\end{array}$ & $\mathbf{2 , 1}$ & $\mathbf{2}$ & $\mathbf{2}$ & $\mathbf{2}$ \\
\hline
\end{tabular}

Fuente: Observatorio meteorológico propio (Villena-Ciudad). Elaboración propia.

* En días.

de marzo y mayo se han contabilizado un mismo número absoluto de rachas, en concreto 4 para ambos, éstas no han agrupado a un idéntico número de días de lluvia; Marzo agrupa a 14 y mayo a 18 días. Por tanto, una vez calculada la longitud media de la racha para ambos meses, se comprueba que la lluvia ha sido un fenómeno más persistente en el mes de mayo, ya que la longitud de las secuencias de lluvia ha sido por término medio de 4,5 días consecutivos registrando precipitación, frente a los 3,5 días del mes de marzo. Por otro lado, si el número de días de lluvia es idéntico en ambos meses - como sucede en los meses de marzo y abril, con 14 días - , pero éstos se agrupan en distinto número de rachas, como ocurre en este ejemplo (marzo en 4 y abril en 6 rachas), la persistencia de los días lluviosos también difiere entre ambos. En este caso, se puede afirmar que el fenómeno de la lluvia resultó más persistente en marzo (longitud media de 3,5 días consecutivos con lluvia) que en el mes de abril (longitud media de 2,33 días).

Por término medio, la longitud media de las secuencias lluviosas en Villena durante la primavera de 2004 fue de 3,29 días (vid. Tabla 3). No obstante, este dato no debe utilizarse como representativo del comportamiento de las rachas lluviosas durante todo el mes, puesto que como puede comprobarse en los datos presentados y los comentarios que se incluyen a continuación, la longitud máxima y mínima de las mismas resulta muy dispar. 


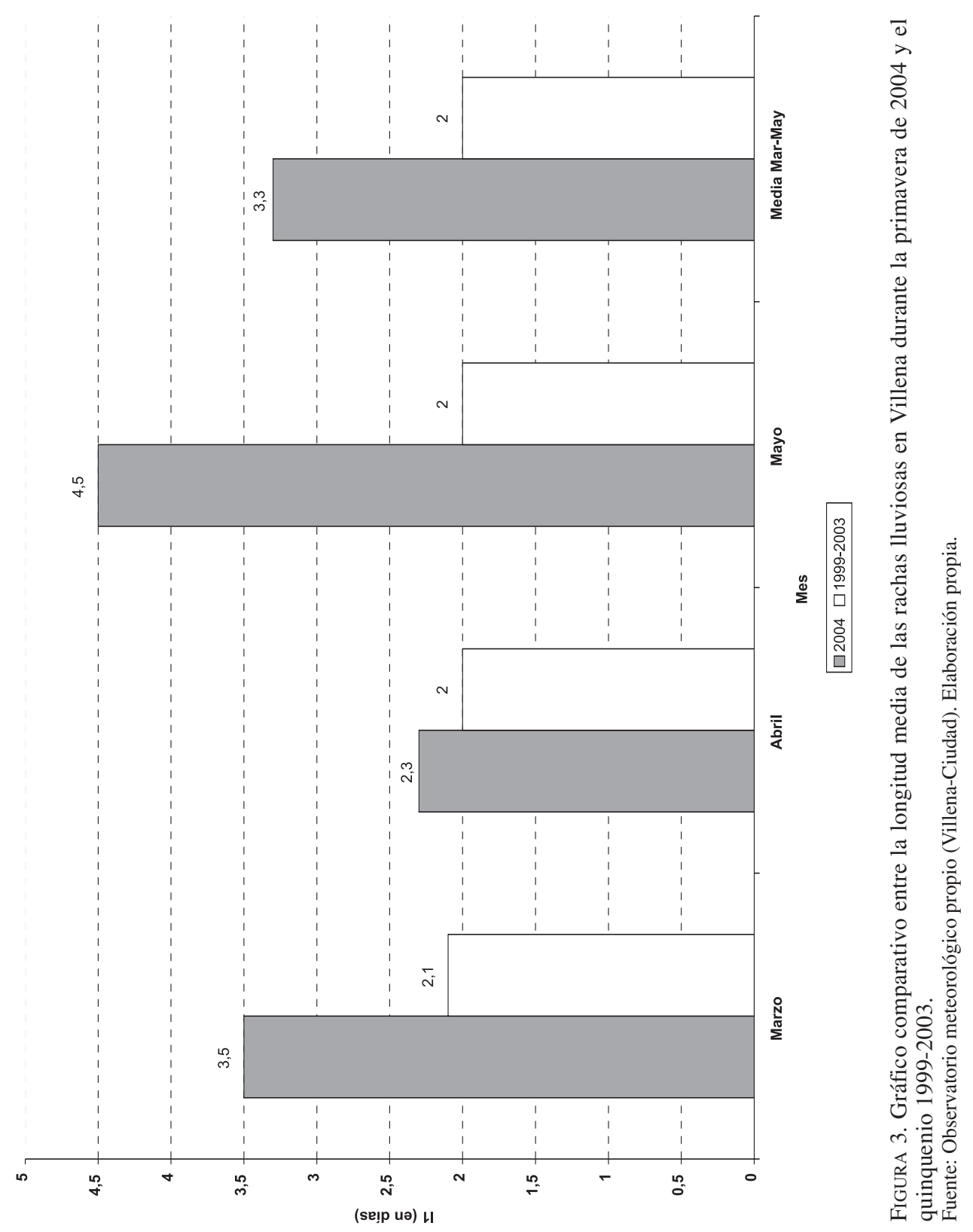


El análisis comparativo de estos datos con los presentados en la tabla 4, que corresponden al cálculo de la longitud media de las rachas lluviosas durante el quinquenio 1999-2003, confirma estadísticamente la persistencia de las jornadas con lluvia durante la primavera de 2004 en Villena. Así, la longitud media calculada de las secuencias lluviosas para los meses de marzo, abril y mayo de 2004 supera para cada uno de ellos la media quinquenal correspondiente a cada mes (vid. Figura 3): marzo $l_{1} 2004=3,5\left(l_{1} 1999-2003=\right.$ $2,1)$; Abril $l_{l} 2004=2,33\left(l_{l} 1999-2003=2\right)$ y mayo $l_{l} 2004=4,5\left(l_{1} 1999-2003=2\right)$. En consecuencia, la longitud media de las rachas lluviosas para la primavera de 2004, de 3,29 días, supera a la obtenida para el quinquenio primaveral 1999-2003, estimada en 2 días; Es decir, la persistencia de los días lluviosos en Villena durante la primavera de 2004 ha sido, por término medio, 1,29 días superior en comparación a la media obtenida para el quinquenio 1999-2003.

Por último, en la tabla 2 se ha incluido también la longitud máxima y mínima absoluta de las rachas lluviosas para los meses de primavera de 2004. Este dato remarca el estado permanente de los días lluviosos en Villena, puesto que las secuencias máximas registrando precipitación en forma de lluvia se elevó a 7 días consecutivos en marzo, 9 días en abril y 5 días en mayo. Estos datos son muy significativos, ya que demuestran la inercia a persistir de las condiciones atmosféricas inestables, de tiempo perturbado y muy lluvioso.

\subsection{Análisis de coeficientes de persistencia de los días lluviosos}

La aplicación de coeficientes de persistencia a la precipitación resulta extraordinariamente interesante a la hora de valorar el carácter repetido del hidrometeoro de la lluvia en Villena durante la primavera de 2004, así como también es de un gran interés para ser aplicado con fines de predicción meteorológica (Martín Vide, 2003). Los cálculos realizados y los resultados obtenidos confirman la elevada persistencia de los días lluviosos en los meses primaverales de marzo, abril y mayo.

Tabla 5

COEFICIENTES DE PERSISTENCIA* DE LOS DÍAS LLUVIOSOS EN VILLENA DURANTE LA PRIMAVERA DE 2004 Y EL QUINQUENIO 1999-2003

\begin{tabular}{|c|c|c|c|c|}
\hline Año & $\boldsymbol{p}_{\boldsymbol{1}}$ & $\boldsymbol{p}_{\boldsymbol{0}}$ & $\boldsymbol{p}_{\boldsymbol{1 1}}$ & $\boldsymbol{p}_{\boldsymbol{1 1}} \boldsymbol{p}_{\boldsymbol{1}}$ \\
\hline $\mathbf{1 9 9 9}$ & 17 & 83 & 38 & 21 \\
\hline $\mathbf{2 0 0 0}$ & 32 & 68 & 45 & 13 \\
\hline $\mathbf{2 0 0 1}$ & 25 & 75 & 35 & 10 \\
\hline $\mathbf{2 0 0 2}$ & 39 & 61 & 66 & 27 \\
\hline $\mathbf{2 0 0 3}$ & 28 & 72 & 54 & 26 \\
\hline $\begin{array}{c}\text { Med. 1999- } \\
\mathbf{2 0 0 3}\end{array}$ & $\mathbf{2 8}$ & $\mathbf{7 2}$ & $\mathbf{5 0}$ & $\mathbf{2 2}$ \\
\hline $\mathbf{2 0 0 4}$ & $\mathbf{5 0}$ & $\mathbf{5 0}$ & $\mathbf{7 0}$ & $\mathbf{2 0}$ \\
\hline
\end{tabular}

$p_{1}$ : probabilidad de día de lluvia $p_{0}$ : probabilidad de día sin lluvia $p_{11}$ : probabilidad de día de lluvia después de día de lluvia

$\boldsymbol{p}_{11-} \boldsymbol{p}_{1}$ : índice de persistencia de los días lluviosos $p_{l 1}>p_{l}$

Fuente: Observatorio meteorológico propio (Villena-Ciudad). Elaboración propia.

$*$ En $\%$. 


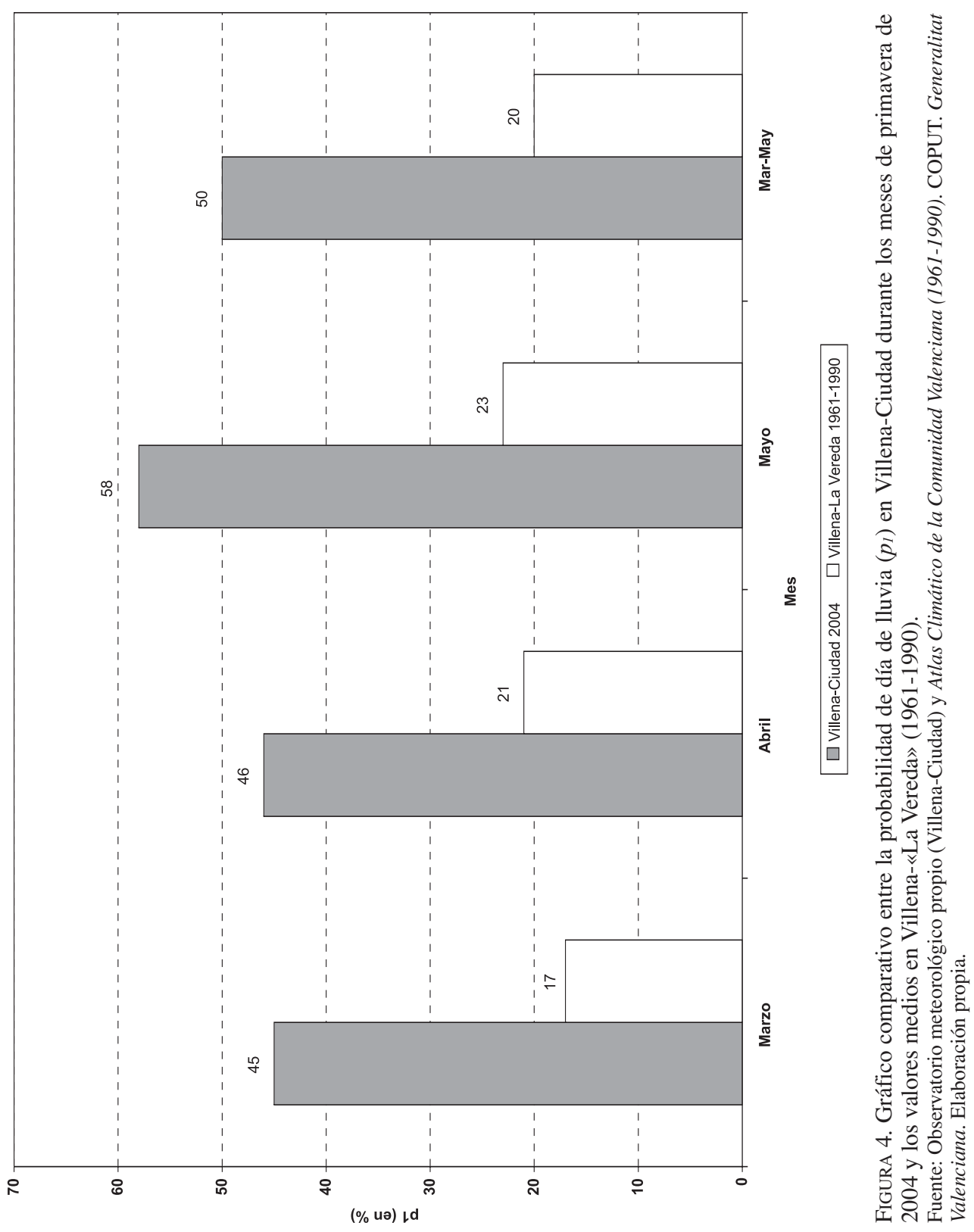


La probabilidad de día lluvioso $\left(p_{l}\right)$ calculada para la primavera de 2004, del 50\%, es reveladora del estado meteorológico de inestabilidad atmosférica y lluvia que caracterizó a esta época del año en toda la geografía alicantina. Es decir, 1 de cada 2 días del período transcurrido entre marzo y mayo se registró precipitación, probabilidad que se sitúa muy por encima de la media calculada para el quinquenio 1999-2003, del 28\% (algo menos de 1 día de cada 3). Lógicamente, la probabilidad de día sin lluvia $\left(p_{0}\right)$ es también del $50 \%$, inferior a la media para los meses de primavera de 1999-2003, del $72 \%$.

El manejo de probabilidades condicionadas, en este caso la probabilidad de día de lluvia después de día de lluvia, afina la estimación del carácter persistente de los días de precipitación. En consecuencia, el valor obtenido para la primavera de 2004, del 70\%, es superior a la media quinquenal de 1999-2003, del 50\%. No obstante, el dato que reafirma la persistencia de los días lluviosos y confirma estadísticamente el estado de inercia de los días de lluvia se obtiene comparando este valor $\left(p_{l l}\right)$ con el de probabilidad de día lluvioso $\left(p_{l}\right)$ : si se cumple la regla de que $p_{l l}>p_{l}$ se corrobora la persistencia de los días con precipitación. Como se observa en la última columna de la derecha de la tabla $5, p_{I l}$ es mayor a $p_{I}$ para todos los casos; En los meses de primavera de 2004, $p_{11}(70 \%)$ es mayor a $p_{1}(50 \%)$.

\section{Conclusión}

La metodología empleada en esta nota ha servido para calcular el grado de persistencia de los días lluviosos en Villena (Alicante) durante los meses de primavera de 2004. Los resultados obtenidos confirman la percepción de la población de haber vivido una primavera extraordinariamente lluviosa, no sólo en cuanto a volúmenes de agua precipitada, verdaderamente extraordinarios, sino al estado repetido de las jornadas de inestabilidad atmosférica y, en consecuencia, de los días lluviosos.

\section{Agradecimientos}

A mis hermanos José Antonio y Juan Carlos, por su dedicación en las tareas de observación meteorológica en la estación de Villena-Ciudad. Al Dr. Javier Martín Vide, Catedrático de Geografía Física en la Universidad de Barcelona, por sus valiosas enseñanzas en materia de métodos de análisis probabilístico aplicados al estudio del clima. El autor disfruta de la beca predoctoral FPU del Ministerio de Educación y Ciencia, que desarrolla en el marco de las actividades del Instituto Universitario de Geografía de la Universidad de Alicante y de las estructuras de investigación del Grup de Climatologia de la Universidad de Barcelona.

\section{Referencias bibliográficas}

BARRIENDOS VALLVÉ, M. y MARTÍN VIDE, J. (1996): «Aplicación metodológica de procesos markovianos a series documentales de ocurrencia diaria de la precipitación en Barcelona (siglos XVII-XVIII», en Clima y agua: la gestión de un recurso climático (Marzol, M.V., Dorta, P., Valladares, P., eds.), Universidad de La Laguna, Tenerife, pp. 261-270.

CONESA GARCÍA, C. y MARTÍN VIDE, J. (1993): «Analyse par la chaine de Markov de la sécheresse dans le sud-est de 1’Espagne», en Rev. Sécheresse, n. ${ }^{\circ}$ 2, vol. 4, pp. 123 129.

DOMíNGUEZ, J. I. (1973): Las cadenas de Markov, su aplicación al clima de Málaga. Departamento de Estadística. Universidad de Málaga. 
DOUGUÉDROIT, A. (1987): «The variations of dry spells in Marseilles from 1865 to 1984», Journal of Climatology, n. ${ }^{\circ}$ 7, pp. 541-551.

FERNÁNDEZ DE ARRÓYABE HERNÁEZ, P. (2002): «La persistencia de la precipitación en forma de lluvia como indicador relativo del cambio climático», en Guijarro, Grimalt, Laita y Alonso, El Agua y el Clima, publicaciones de la AEC, serie A, n. ${ }^{\circ} 3$, Mallorca, pp. 199-207.

GIL OLCINA, A. y MORALES GIL, A. (Eds.), (2001): Causas y consecuencias de las sequías en España, Caja de Ahorros del Mediterráneo e Instituto Universitario de Geografía de la Universidad de Alicante, Alicante, $574 \mathrm{pp}$.

GÓMEZ NAVARRO, L. (1996): «Análisis de las secuencias lluviosas en España mediante el modelo de las cadenas de Markov», en Ciudad y clima: la gestión de un recurso climático (Marzol, M.V., Dorta, P., Valladares, P., eds.), Universidad de La Laguna, Tenerife.

GÓMEZ NAVARRO, L. (1997): Regionalización climática de la España peninsular mediante el análisis Markoviano de las sequías. Tesis doctoral.

GÓMEZ NAVARRO, L., MARTÍN VIDE, J. y WANNER, H. (2000): «Regionalización de la España peninsular basada en la longitud de las secuencias lluviosas (1951-1990)», en Riegos y Drenajes XXI, pp. 38-45.

LANA, X., BURGUEÑO, A. (1998): Daily dry-wet behaviour in Catalonia (NE Spain) from the viewpoint of Markov chains, International Journal of Climatology, n. ${ }^{\circ} 18$, pp. 793-815.

LUENGO UGIDOS, M. A., CEBALLOS BARBANCHO, A., MARTÍNEZ FERNÁNDEZ, J. y YUSTE YUSTE, C. (2002): «Las rachas secas en el sector central de la cuenca del Duero», en Investigaciones Geográficas, n. ${ }^{\circ}$ 27, Instituto Universitario de Geografía, Universidad de Alicante, Alicante, pp. 65-82.

MARTÍN VIDE, J. (1981): «Cantidades diarias y análisis markoviano de las precipitaciones en el litoral mediterráneo sur de la Península Ibérica», en Paralelo 37, n. ${ }^{\circ}$ 5, pp. 97-114.

MARTÍN VIDE, J. (1983): «La aceptación del modelo estocástico de la cadena de Harkov homogénea en tiempo discreto y de dos estados en los cálculos de la probabilidad de la precipitación diaria», en VIII Congreso de Geógrafos Españoles. Comunicaciones, Barcelona, AGE, pp. 24-31.

MARTÍN VIDE, J. y MORENO GARCÍA, M.C. (1985): «El estudio de las sequías mediante el análisis probabilística de las secuencias secas. El caso de Almería», en IX Coloquio de Geografía. Ponencias I, AGE.

MARTÍN VIDE, J., BIELSA, I., GARCÉS, M. P., MÓDENES, J. A., OLIVAS, A., PÉREZ, J., RUCABADO, M., SALVADOR, M. y SÁNCHEZ, E. (1989): «La bondad de la cadena de Markov de primer orden en el cálculo de la probabilidad de las secuencias lluvias y secas en Catalunya», en Notas de Geografía Física, n. ${ }^{\circ}$ 18, pp. 51-57.

MARTÍN VIDE, J. (2003): El tiempo y el clima, Editorial Rubes, Barcelona, 127 pp.

MARTÍN VIDE, J. (2004): «Spatial distribution of a daily precipitation concentration index in peninsular Spain», International Journal of Climatology, $\mathrm{n}^{\circ} 24$, pp. 959-971.

PÉREZ CUEVA, A. J. (Coord.), (1994): Atlas climático de la Comunidad Valenciana (1961-1990), Generalitat Valenciana, Conselleria d'Obres Publiques, Urbanisme i Transports, Valencia, $208 \mathrm{pp}$.

RASO NADAL, J. M. (1982): «Probabilidades de transición y distribución estacionaria de los días con y sin precipitación en Palma de Mallorca según el modelo de las cadenas de Markov para dos estados», Tarraco, nº 3, pp. 195-209. 


\section{ANEXO}

Tabla 6

FICHA DE ANOTACIÓN DIARIA DE PRECIPITACIÓN, CON INDICACIÓN DEL VIENTO DOMINANTE Y METEOROS OBSERVADOS. VILLENA (ALICANTE), MARZO, ABRIL Y MAYO DE 2004

\begin{tabular}{|c|c|c|c|c|c|c|}
\hline & \multirow{2}{*}{ Día } & \multirow{2}{*}{$\begin{array}{l}\text { Precipitación } \\
\text { (mm) }\end{array}$} & \multicolumn{3}{|c|}{ Meteoros observados } & \multirow{2}{*}{$\begin{array}{c}\text { Viento } \\
\text { dominante }\end{array}$} \\
\hline & & & $\mathbf{M}$ & $\mathbf{T}$ & $\mathbf{N}$ & \\
\hline \multirow[t]{31}{*}{ Marzo } & 1 & & & & & \\
\hline & 2 & & & & & \\
\hline & 3 & & & & & \\
\hline & 4 & & & & & \\
\hline & 5 & & & & & \\
\hline & 6 & ip. & $\bullet / 9$ & $\bullet 19$ & & ONO \\
\hline & 7 & & & & & \\
\hline & 8 & & & & & \\
\hline & 9 & & & & & \\
\hline & 10 & ip. & $\bullet / 9$ & & & $\mathrm{NO}$ \\
\hline & 11 & 0,3 & $\bullet / 9$ & & & ONO \\
\hline & 12 & & & & & \\
\hline & 13 & 4,3 & $\bullet$ & & & $\mathrm{SSO}$ \\
\hline & 14 & 1,2 & $\bullet 19$ & & & $\mathrm{NE}$ \\
\hline & 15 & ip. & 9 & 9 & 9 & $\mathrm{NE}$ \\
\hline & 16 & 1,5 & $\bullet 19$ & & & NE \\
\hline & 17 & & & & & \\
\hline & 18 & & & & & \\
\hline & 19 & & & & & \\
\hline & 20 & & & & & \\
\hline & 21 & & & & & \\
\hline & 22 & & & & & \\
\hline & 23 & & & & & \\
\hline & 24 & & & & & \\
\hline & 25 & 6,0 & & - 19 & $\bullet / 9$ & $\mathrm{NE}$ \\
\hline & 26 & 20,1 & $\bullet / 9$ & - 19 & $6 / 9$ & $\mathrm{NE}$ \\
\hline & 27 & 1,9 & $\bullet / 9$ & - 19 & 019 & NE \\
\hline & 28 & 0,4 & $\bullet / 9$ & & - 19 & $\mathrm{NE}$ \\
\hline & 29 & 23,5 & $\bullet / 9$ & & & $\mathrm{NE}$ \\
\hline & 30 & 1,1 & $\bullet / 9$ & $\bullet 19$ & & ENC. \\
\hline & 31 & ip. & & $\bullet 19$ & & ONO \\
\hline \multirow[t]{22}{*}{ Abril } & 1 & & & & & \\
\hline & 2 & 0,7 & $\bullet / 9$ & & & $\mathrm{O}$ \\
\hline & 3 & & & & & \\
\hline & 4 & & & & & \\
\hline & 5 & & & & & \\
\hline & 6 & & & & & \\
\hline & 7 & 0,4 & & $\bullet / 9 / R$ & & SE \\
\hline & 8 & ip. & $\bullet / 9$ & & & $\mathrm{NE}$ \\
\hline & 9 & 0,5 & $\bullet / 9$ & & & $\mathrm{NE}$ \\
\hline & 10 & 3,4 & 019 & $\bullet 19$ & & NE \\
\hline & 11 & 8,5 & 019 & 9 & & NNE \\
\hline & 12 & 0,2 & & 019 & & $\mathrm{NO}$ \\
\hline & 13 & & & & & \\
\hline & 14 & & & & & \\
\hline & 15 & 6,0 & & $\bullet / 9$ & -19 & $\mathrm{NE}$ \\
\hline & 16 & 23,4 & $\bullet / 9$ & $\bullet / 9$ & & VAR. \\
\hline & 17 & & & & & \\
\hline & 18 & ip. & & & 9 & ONO \\
\hline & 19 & & & & & \\
\hline & 20 & & & & & \\
\hline & 21 & & & & & \\
\hline & 22 & ip. & & $\bullet$ & & $\mathrm{NO}$ \\
\hline
\end{tabular}




\begin{tabular}{|c|c|c|c|c|c|c|}
\hline & 15 & 6,0 & & $\cdot 19$ & - 19 & $\mathrm{NE}$ \\
\hline & 16 & 23,4 & $\cdot 19$ & $\cdot 19$ & & VAR. \\
\hline & 17 & & & & & \\
\hline & 18 & ip. & & & 9 & ONO \\
\hline & 19 & & & & & \\
\hline & 20 & & & & & \\
\hline & 21 & & & & & \\
\hline & 22 & ip. & & - & & NO \\
\hline & 23 & & & & & \\
\hline & 24 & & & & & \\
\hline & 25 & & & & & \\
\hline & 26 & & & & & \\
\hline & 27 & & & & & \\
\hline & 28 & 8,4 & & $\cdot 19$ & $\cdot 19$ & NNE \\
\hline & 29 & ip. & $\cdot 19$ & & & NNE \\
\hline & 30 & ip. & $\cdot 19$ & & & ONO \\
\hline \multirow[t]{31}{*}{ Mayo } & 1 & 4,5 & & $\cdot / 9 / R$ & $\bullet / R$ & $\mathrm{O}$ \\
\hline & 2 & 4,0 & & $\cdot 19$ & $\bullet 19$ & $\mathrm{NE}$ \\
\hline & 3 & 11,1 & $\bullet 19$ & $\cdot 19$ & - 19 & $\mathrm{NE}$ \\
\hline & 4 & 3,4 & $\bullet 19$ & & & $\mathrm{NNO}$ \\
\hline & 5 & ip. & & $\cdot 19$ & & ONO \\
\hline & 6 & 0,7 & $\bullet 19$ & & & $\mathrm{NO}$ \\
\hline & 7 & & & & & \\
\hline & 8 & & & & & \\
\hline & 9 & & & & & \\
\hline & 10 & 12,1 & & $\cdot 19 / 6$ & 9 & VAR. \\
\hline & 1 & 18,5 & 019 & $\bullet / 9$ & $\cdot 19$ & $\mathrm{NE}$ \\
\hline & 12 & 1,1 & $\bullet 19$ & & & $\mathrm{~N}$ \\
\hline & 13 & 1,5 & & $\bullet / 9 / R$ & & VAR. \\
\hline & 14 & ip. & $\bullet 19$ & $\bullet / 9$ & & $\mathrm{NE}$ \\
\hline & 15 & & & & & \\
\hline & 16 & & & & & \\
\hline & 17 & & & & & \\
\hline & 18 & & & & & \\
\hline & 19 & & & & & \\
\hline & 20 & & & & & \\
\hline & 21 & ip. & & - 19 & & $\mathrm{O}$ \\
\hline & 22 & 1,1 & -19 & - 19 & & SE \\
\hline & 23 & ip. & & - 19 & & $\mathrm{E}$ \\
\hline & 24 & & & & & \\
\hline & 25 & 0,4 & & $\bullet 19$ & & SE \\
\hline & 26 & ip. & & $\cdot / 9 / \mathrm{R}$ & & $\mathrm{SE}$ \\
\hline & 27 & 3,8 & & $\cdot 19 / \mathrm{R}$ & & SE \\
\hline & 28 & & & & & \\
\hline & 29 & ip. & & $\cdot 19 / \mathrm{R}$ & & SE \\
\hline & 30 & & & & & \\
\hline & 31 & & & & & \\
\hline
\end{tabular}

Fuente: Archivo de datos climáticos del observatorio meteorológico propio (Villena-Ciudad).

\section{Rachas o secuencias lluviosas}

\section{Meteoros}

- Lluvia

9 Llovizna

R Tormenta 